\title{
RePro-Manager, Transaktionskosten und nachhaltige Wertschöpfung
}

\author{
Die Nutzung von Sekundärressourcen aus der \\ Energie-, Wasser-, Land- und Forstwirtschaft \\ verspricht im Spannungsfeld der Landnutzung \\ sowohl wirtschaftlichen Erfolg als auch ökolo- \\ gische Nachhaltigkeit bei der Energieproduktion. \\ Wie können solche Wertschöpfungsketten \\ aufgebaut und Hemmnisse bewältigt werden? \\ Von Özgür Yildiz und Susanne Schön
}

$\mathbf{R}$ egionale Re-Produktionsketten verheißen wirtschaftlich und ökologisch tragfähige Entwicklung gleichermaßen, sind jedoch im Zuge ihrer Realisierung verschiedenen Hemmnissen ausgesetzt. Dennoch ist es im Rahmen des Forschungsvorhabens „RePro - Ressourcen vom Land“ (RePro) gelungen, vereinzelt Wertschöpfungsketten erfolgreich zu initiieren. Dieser Beitrag reflektiert die Aufbauprozesse im Spiegel von Erkenntnissen aus Innovationsforschung und Neuer Institutionenökonomik.

\section{Nachhaltige Wertschöpfung in schrumpfenden Regionen?}

Ausgangspunkt des vom Bundesministerium für Bildung und Forschung (BMBF) geförderten dreijährigen Forschungsprojekts RePro war die Frage, wie man in schrumpfenden Regionen neue, nachhaltige Wertschöpfungsketten tragfähig umsetzen kann. Denn zurückgehende Bevölkerungszahlen heißen auch schrumpfende Märkte, Verlust an unternehmerischem Potenzial und Kapital sowie eine geringere Innovationsdynamik.

Die erste These zu dieser Frage lautete: Möglicherweise ist die Energie- und Wasserinfrastruktur ein guter Ansatzpunkt für neue Wertschöpfungsketten, weil man an einer funktionierenden Organisation mit einer dauerhaften Aufgabe andocken kann. Zudem sind diese beiden Infrastrukturbereiche durch den Klimawandel, die Energiewende und den Bevölkerungsrückgang unter Innovationsdruck und damit in Bewegung geraten. Auch die zweite These ging von der Überlegung aus, dass man am besten mit dem arbeitet, was vor Ort noch vorhanden ist. Damit gerieten regionale Rest- und Abfallstoffe, die als Sekundärressourcen verwertet werden könnten, in den Blick.

Aufgabe war es nun, mit der regionalen Verwertung von Sekundärressourcen nachhaltige Wertschöpfungsketten in der Energie- und Wasserinfrastruktur zu identifizieren und exemplarisch aufzubauen. „Nachhaltig“ haben die Forscher von RePro in Anlehnung an das (Re)Produktivitätskonzept von Biesecker/Hofmeister (2006) so übersetzt, dass die Wertschöpfungsketten sowohl die stofflich-energetische Re-Produktionsfähigkeit als auch die wirtschaftlich-soziale Re-Produktionsfähigkeit der Regionen erhalten oder verbessern sollen. Solche Wertschöpfungsketten werden daher Re-Produktionsketten oder kurz „RePro-Ketten“ genannt.

\section{RePro-Ketten und der RePro-Planer}

Das Forschungsteam des Vorhabens hat sieben solcher RePro-Ketten identifiziert, die unter heutigen wirtschaftlichen, technischen und rechtlichen Bedingungen umsetzbar sind (Abbildung 2). In diesen Wertschöpfungsketten werden Sekundärressourcen wie Grünschnitt, Waldrestholz, Biogasanlagen-Abwärme, gereinigtes Abwasser, Klärschlamm und bislang nicht nutzbare Flächen zu Produkten wie Strom, Wärme, Gas, Düngemittel oder Energieholz verarbeitet.

Drei RePro-Ketten befinden sich in den beiden Modellregionen Landkreis Elbe-Elster (Brandenburg) und Landkreis Wittenberg (Sachsen-Anhalt) derzeit in unterschiedlichen Stadien der Umsetzung.

Darüber hinaus hat das Forschungsteam umfangreiche Informations- und Arbeitsmaterialien erstellt, mit deren Hilfe auch in anderen Regionen RePro-Ketten aufgebaut werden können. Der RePro-Planer, so die Bezeichnung dieses Werkzeugkastens (Abbildung 1), richtet sich an interessierte Laien

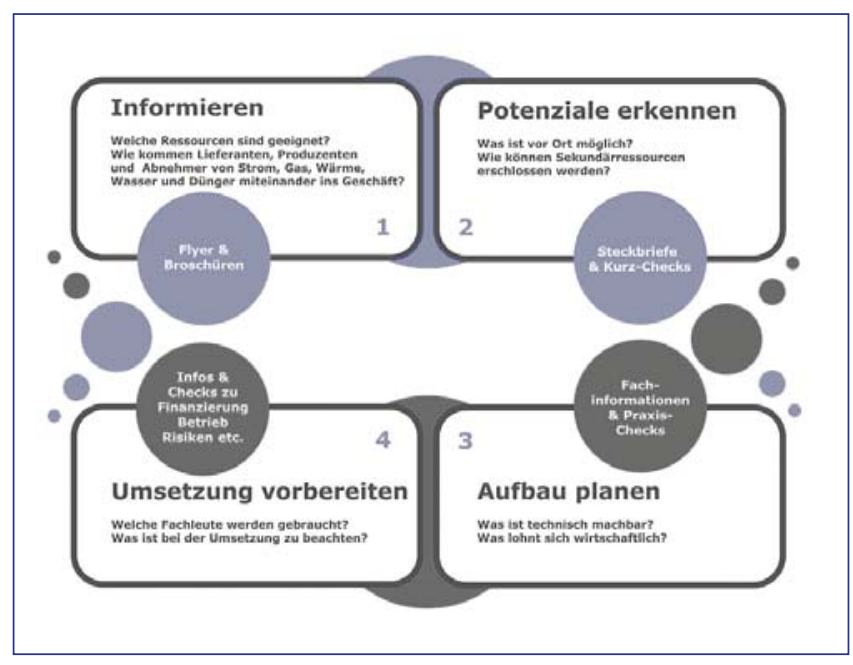

Abbildung 1: Der RePro-Planer in der Übersicht 
mit Halbwissen: Bürgermeister, Wirtschaftsförderer, Kreisund Regionalentwickler, regionale Initiativen und Unternehmen etc., die die Idee der RePro-Ketten aufgreifen, initiativ werden und den Aufbauprozess vorantreiben wollen. [1]

\section{RePro-Ketten und} regionale Wertschöpfung

Ob die RePro-Ketten über die beiden Modellregionen hinaus ein Erfolgsmodell werden, hängt allerdings nicht nur von einem gelungenen Informations- und Wissenstransfer ab. Vielmehr erfordern RePro-Ketten eine koordinierte Zusammenarbeit von mehreren Akteuren, die in der Regel noch keine Wirtschaftsbeziehungen zueinander unterhalten und die noch dazu in verschiedenen Sektoren und auf verschiedenen Ebenen angesiedelt sind, sodass unterschiedliche Interessen aufeinandertreffen: zum Beispiel land- und forstwirtschaftliche Betriebe, Abwasserentsorger und Energieversorger, Flächeneigentümer und Kommunen, Bürger und Unternehmen. Der Aufbau von RePro-Ketten verlangt ihnen $\mathrm{ab}$, dass sie sich mit der Idee auseinandersetzen, sich gegebenenfalls zu Investitionsentscheidungen durchringen und in Verträge oder Organisationen einbinden lassen.

Warum sollten sie in einem wenig standardisierten und etablierten Bereich wirtschaftliche Risiken eingehen? Was gibt es für sie zu gewinnen? Wie kann das konkret vonstattengehen? Kann man den anderen trauen? Was, wenn ein Partner aussteigt oder in Insolvenz geht?

Diese und weitere berechtigte Fragen gilt es zu beantworten, um eine belastbare Informationsbasis für die notwendigen Entscheidungen zu schaffen, um Vertrauen zwischen den Beteiligten wachsen $\mathrm{zu}$ lassen und um individuelle und gemeinsame Interessen für alle tragbar auszutarieren. Das geht nicht von heute auf morgen. Es ist ein langer Weg, auf dem Zeit, Engagement, Beständigkeit und Kompetenz gebraucht werden. Zwei für die Zukunft der RePro-Ketten entscheidende Fragen lauten daher:

-Wer kann diese Informations- und Koordinationsleistungen erbringen?

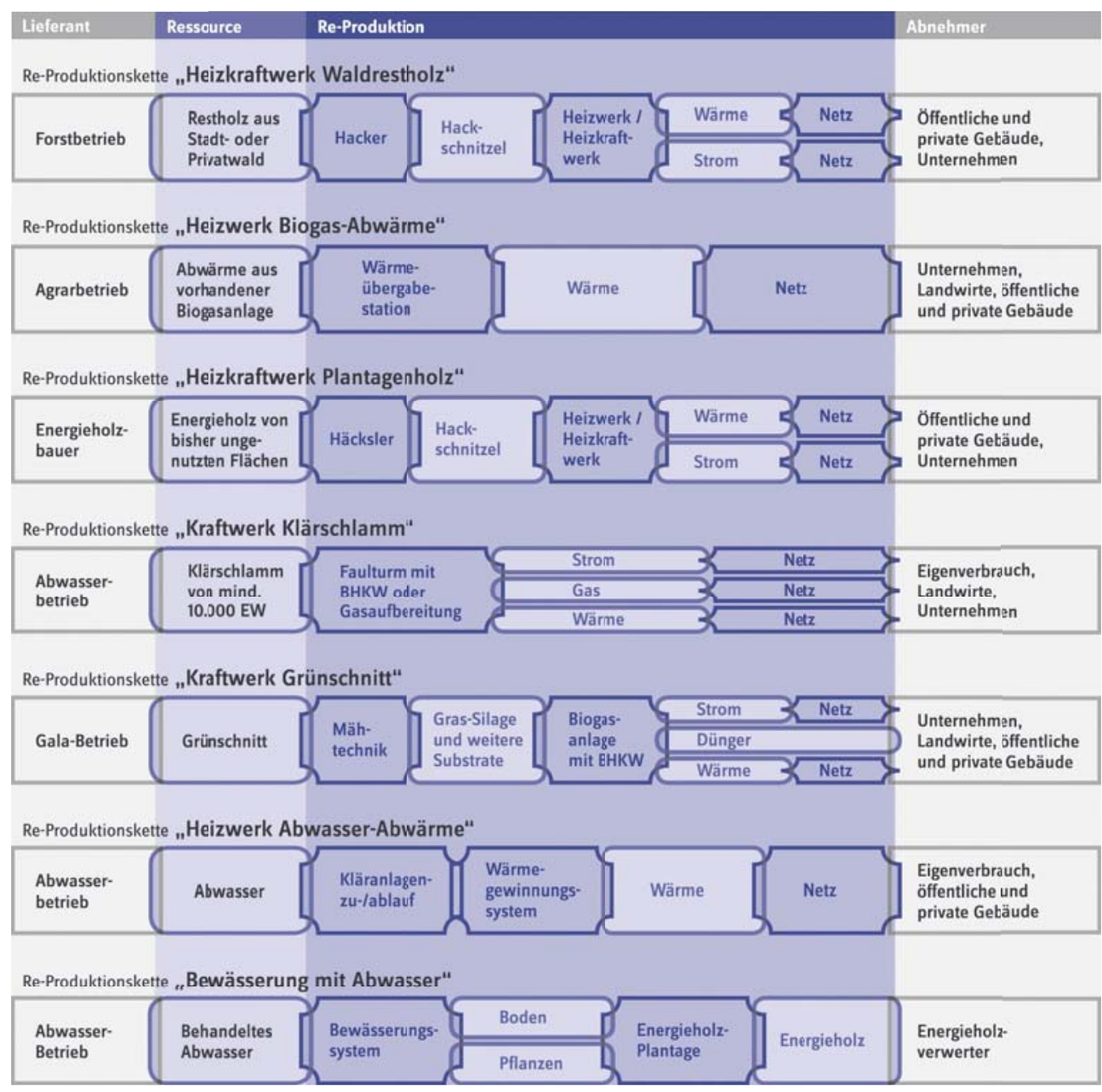

Quelle: eigene Darstellung
- Wie können die Kosten, die durch die geschilderte, vergleichsweise aufwendige Konstruktion der RePro-Ketten entstehen, gemindert und/oder getragen werden?

\section{Der RePro-Manager als Erfolgs- bedingung}

Es ist unbestritten, dass es eine Art RePro-Manager geben muss, der beim Aufbau einer RePro-Kette die Fäden in die Hand nimmt und in der Hand behält. Auch in den beiden RePro-Modellregionen wäre es trotz motivierter Akteure nicht ohne ein koordinierendes Management gegangen. Allerdings hatten sie gegenüber den potenziellen Nachahmer-Regionen einen großen Vorteil: Die Förderung des BMBF ermöglichte es jeder Region, für die Dauer von drei Jahren einen Regionalkoordinator zu beschäftigen, der sich ausschließlich dem Vorantreiben der RePro-Ketten widmete.

Die folgende Ausführungen und Grafiken (siehe Abbildung 3 und Abbildung 4) veranschaulichen die wichtigsten Elemente und Tätigkeitsbeschreibungen in einer bestimmten Aufbauphase verschiedener RePro-Ketten. 
Tätigkeitsschwerpunkte des RePro-Managers

Das erste Fallbeispiel bildet eine Übersicht der beteiligten Akteure, der erforderlichen Ressourcen und technischen Einheiten der kombinierten RePro-Ketten Heizwerk Biogasabwärme und Heizkraftwerk Waldrestholz in der brandenburgischen Stadt Uebigau-Wahrenbrück ab. Hier sollen Hackschnitzel aus Waldrestholz und die Abwärme aus einer Biogasanlage für die Wärmeversorgung zweier Stadtteile genutzt werden. Mit dem noch $\mathrm{zu}$ errichtenden Nahwärmenetz können 100 Haushalte mit Wärme versorgt werden. Ohne in die Details der Grafik einzutauchen, soll sie einen beispielhaft pointierten Eindruck von den Aufgaben und Tätigkeiten des RePro-Managers geben.

Abbildung 3 zeigt verschiedene Elemente-Gruppen, die für durchaus typische Tätigkeitsschwerpunkte des RePro-Managements stehen, das sich in diesem Fall aus dem Bürgermeister, einer Regionalkoordinatorin und einem punktuell hinzugezogenen Forstwirt (Projektkoordinator) zusammensetzt. Tätigkeitsschwerpunkte des RePro-Managements im Rahmen dieses Fallbeispiels waren und sind (von links gegen den Uhrzeigersinn):

- Die Lieferanten und Verarbeiter der Sekundärressourcen wirtschaftlich zusammenzuspannen und zu abgestimmten Investitionsentscheidungen $\mathrm{zu}$ bringen,

- die Einwohner anhand vergleichbarer Erfolgsbeispiele mit der Idee vertraut zu machen, sie immer wieder zu informieren und mit belastbaren Berechnungen von der Sinnhaftigkeit des Vorhabens zu überzeugen,

- die Hausbesitzer, die angeschlossen werden sollen und wollen, $\mathrm{zu}$ informieren und $\mathrm{zu}$ motivieren, zugleich die erforderlichen Daten für die technisch-betriebswirtschaftlichen Analysen zu erheben,

- die perspektivische Einbeziehung weiterer Betriebe, Sekundärressourcen und Abnehmer,

- die Vorbereitung und Umsetzung der Organisationsform Bürgerenergiegenossenschaft sowie der Finanzierung des Nahwärmenetzes.

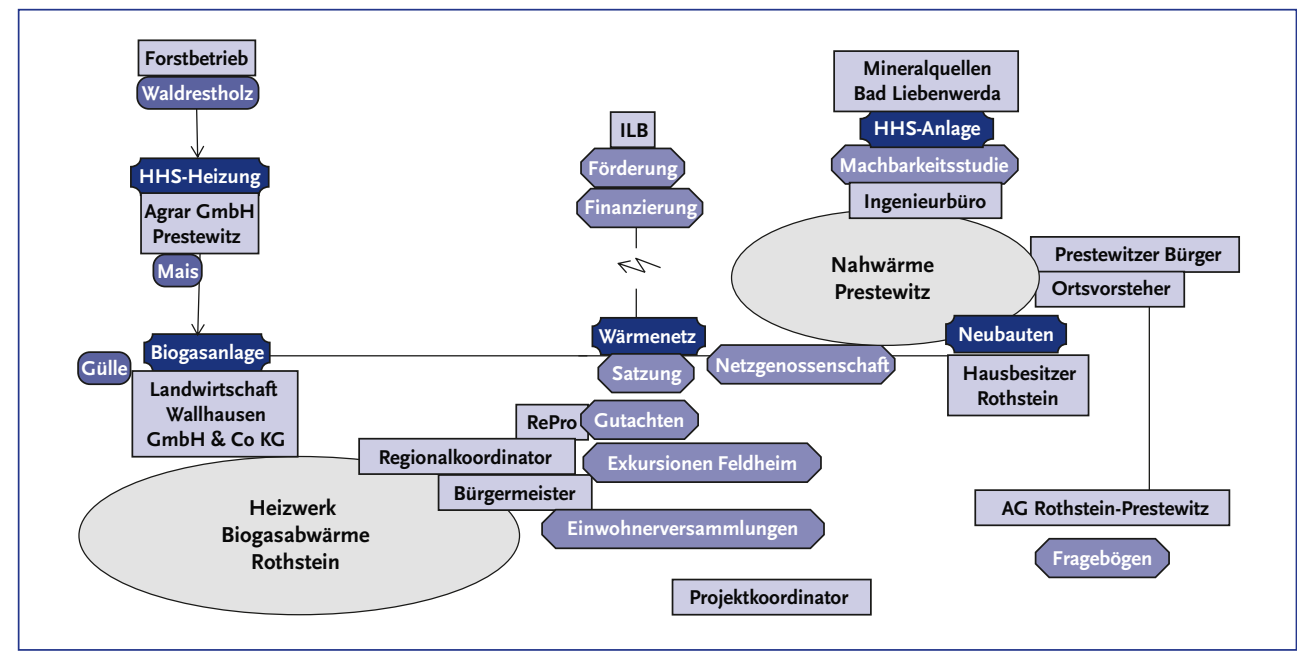

Quelle: eigene Darstellung

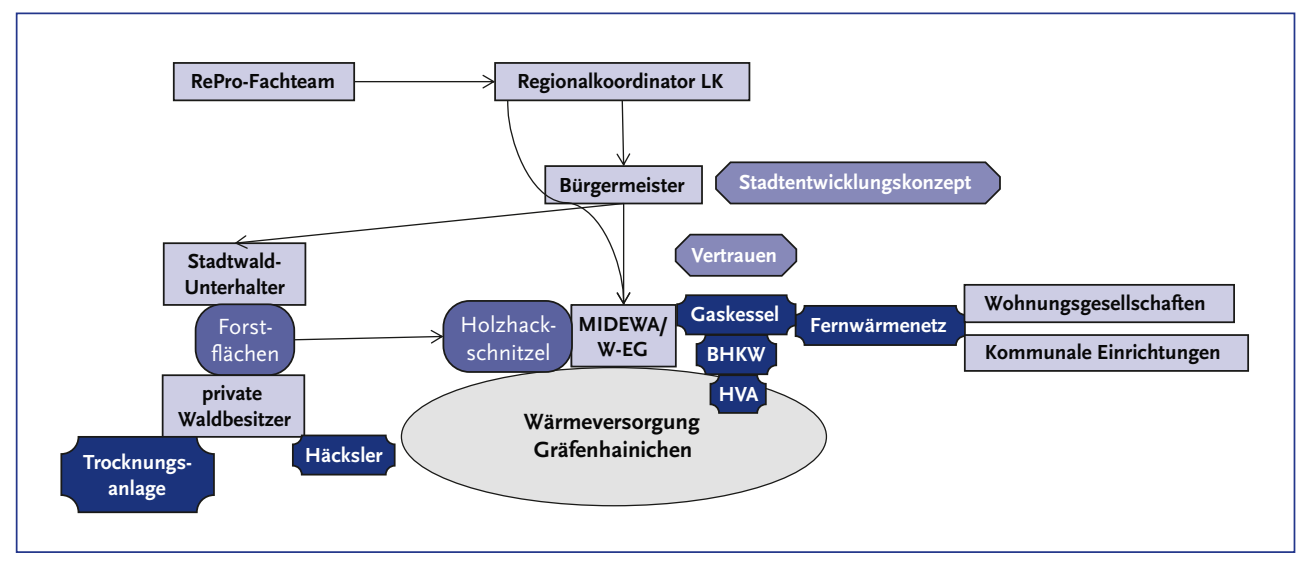

Quelle: eigene Darstellung

Das zweite Fallbeispiel veranschaulicht den Prozess entlang der RePro-Kette Heizkraftwerk Waldrestholz in der Gemeinde Gräfenhainichen im Landkreis Wittenberg. Hier sollen in bestehenden Versorgungsstrukturen fossile Energieträger teilweise durch erneuerbare Energieträger substituiert werden, um Versorgungssicherheit und Preisstabilität zu gewährleisten. Dabei gibt es vor Ort erhebliche Vorbehalte, da vor einigen Jahren ein Palmöl-Blockheizkraftwerk (BHKW) aufgrund der Entwicklungen am Rohstoffmarkt in finanzielle Schwierigkeiten geriet und schließlich abgeschaltet werden musste. Das Vertrauen in neue Versorgungskonzepte musste erst wiedergewonnen werden.

In Analogie zu Abbildung 3 stellen die Elemente in Abbildung 4 zentrale Tätigkeitsschwerpunkte des RePro-Regionalkoordinators in Gräfenhainichen dar. Tätigkeitsschwerpunkte des RePro-Managements im Rahmen dieses Fallbeispiels waren und sind (von links gegen den Uhrzeigersinn):

- Die Abstimmung zwischen dem Bürgermeister und dem Betreiber der öffentlichen Forstflächen (Stadtwald-Unterhalter) zu koordinieren, um die verfügbare Menge an Holz- 
hackschnitzeln aus öffentlichen Flächen und den dazugehörigen Arbeitspreis zu ermitteln,

I moderierend die Preisverhandlungen zwischen Holzhackschnitzel (HHS)-Lieferanten und den Betreibern der Holzverbrennungsanlage (HVA) zu begleiten, dabei durch transparente Informationen zu langfristigen Vereinbarungen zu kommen, die die Interessen aller Parteien berücksichtigen, und zudem Sicherheit für die Investition der privaten Waldbesitzer in eine Trocknungsanlage und des lokalen Energieversorgers (MIDEWA/W-EG) in eine HVA zu schaffen,

I die lokalen Energieabnehmer (Wohnungsbaugesellschaften, kommunale Abnehmer) über die Chancen des neuen Versorgungskonzepts zu informieren, gleichzeitig aktuelle Informationen über die Entwicklung des künftigen Wärmeverbrauchs sowie mögliche Investitionen in Energieeffizienzmaßnahmen seitens der Wohnungsbaugesellschaften zu erheben und dem lokalen Energieversorger zu kommunizieren

I sowie in Folge des gescheiterten BHKW-Projekts verloren gegangenes Vertrauen zwischen lokalem Energieversorger, Bürgermeister und anderen Interessengruppen wiederherzustellen, indem die avisierte RePro-Kette durch das RePro-Fachteam betriebswirtschaftlich geprüft und mit Risiko-aversen Leitplanken versehen wurde. Alle involvierten Akteursgruppen haben hinreichende Informationen zur ökonomischen Beurteilung der Investition.

\section{Merkmale und Fähigkeiten des RePro-Managers}

Diesen Beispielen folgend, lassen sich zusammengefasst vier Hauptaufgabenbereiche des RePro-Managers benennen:

I Idee und Initiative,

I Information, Motivation, Überzeugung der Beteiligten und des Umfelds,

I Kommunikation und Koordination der Beteiligten,

I Planung und Steuerung des Umsetzungsprozesses.

Welche Anforderungen stellen diese Aufgaben an das ReProManagement und wie haben die Modellregionen sie bewältigt? Das RePro-Managementteam in Uebigau-Wahrenbrück erfüllt die Anforderungen für die beispielhaft dargestellte, relativ komplexe RePro-Kette, in der vieles neu entwickelt und gebaut werden muss, durch seine adäquate Zusammensetzung. Der Bürgermeister, der für die beteiligten Wirtschaftspartner und für die Bürger die Rolle des vertrauenswürdigen und verbindlichen Verantwortlichen übernommen hat; die Regionalkoordinatorin, die jederzeit ansprechbar ist und den gesamten Prozess am Laufen hält; der Forstwirt, der - langjähriger Leiter des örtlichen Forstbetriebs - als erfahrener und vertrauenswürdiger Fachmann die Bürger-Arbeitsgruppen leitet.

In der zweiten RePro-Modellregion, im Landkreis Wittenberg, war das RePro-Management zwangsläufig anders aufgestellt. Der in die Region zugezogene Regionalkoordinator wurde von der Leiterin des Fachdienstes Kreisentwicklung nach und nach mit den wichtigsten Akteuren bekannt gemacht und musste sich deren Vertrauen erst erarbeiten. Als Mitarbeiter des
Landkreises war er darüber hinaus von der operativen Ebene weiter entfernt als seine Kollegin in Uebigau-Wahrenbrück. Folglich mussten lokale RePro-Ketten-Initiativen in jedem Fall mit den jeweiligen Bürgermeistern abgestimmt werden, die dann auch die lokalen Akteure zur ersten Kontaktaufnahme ansprachen und den weiteren Prozess quasi als Gewährsleute begleiteten. Das nahm deutlich mehr Zeit in Anspruch und war in der Koordination aufwendiger. Demgegenüber war die kommunikative Reichweite zur Verbreitung der RePro-KettenKonzepte aber auch größer: Der RePro-Manager wirkte deutlich stärker in den Landkreis hinein und darüber hinaus.

Ungeachtet dieser Unterschiede schälten sich bei der teilnehmenden Beobachtung und der Selbstreflexion beider RePro-Managementteams übereinstimmende Aussagen dazu heraus, was einen idealtypischen RePro-Manager auszeichnet:

I Neutralität: Er darf keine eigenen wirtschaftlichen Interessen verfolgen.

- Lokale Verankerung: Er muss bekannt sein oder werden und sich das Vertrauen seines Umfelds erworben haben oder es sich erwerben.

- Unternehmerische Denke: Er muss in unternehmerischen Kategorien denken können.

- Ausgleichende Funktion: Er muss die betriebswirtschaftlichen Interessen einzelner und die Gemeinwohlinteressen der gesamten Gemeinde austarieren.

I Zähigkeit: Er muss mit Ausdauer, Frustrationstoleranz und einem gewissen Biss den Aufbauprozess vorantreiben.

I Charisma: Er muss vom RePro-Konzept überzeugt sein, um die vielen, die es für den Aufbau einer RePro-Kette braucht, überzeugen zu können.

Auch wenn die Regionalkoordinatoren im RePro-Forschungsprojekt in den öffentlichen Verwaltungen angesiedelt waren und die Modellregionen damit auch gut gefahren sind, gibt es doch weitere Möglichkeiten, die hier kurz erörtert werden sollen. Jedes Modell hat dabei Vor- und Nachteile.

Das Neutralitätsgebot, die lokale Verankerung und die Fähigkeit zum Interessenausgleich sprechen für die Verankerung eines RePro-Managers in der öffentlichen Verwaltung. Bürgermeister, Kreisentwickler, Wirtschaftsförderer sind zweifellos gut geeignete Kandidaten. Die Fähigkeit zum unternehmerischen Denken kann (und muss gegebenenfalls) im Laufe des Prozesses entwickelt werden. Zähigkeit und Charisma sind dagegen als personenbezogene Eigenschaften vom konkreten Koordinator abhängig. Da Verwaltungen unter etwas geringerem Effizienz- und Verwertungsdruck stehen als Unternehmen, haben sie gegebenenfalls einen längeren Atem.

Ein professioneller Dienstleister, der als Auftragnehmer eine RePro-Kette aufbaut, ist die zweite Möglichkeit. Auch er würde unter bestimmten Bedingungen das Neutralitätsgebot erfüllen - wenn beispielsweise die Gemeinde oder die an der RePro-Kette Beteiligten Auftraggeber sind - und hätte in Sachen unternehmerischer Denke und ausgleichender Funktion Managementqualitäten. Die lokale Verankerung wäre in diesem Modell durch den Auftraggeber gegeben. Zähigkeit und 
Charisma könnten durch ein erfolgsabhängiges Honorar befördert werden.

Als dritte Variante ist ein Unternehmen denkbar, das vom Zusammenwirken der unterschiedlichen Beteiligten profitiert. In der oben abgebildeten RePro-Kette in Rothstein-Prestewitz könnte das beispielsweise der Betreiber des Nahwärmenetzes sein. Er hätte dann zwar eigene wirtschaftliche Interessen, die aber nur durch das für alle profitable Zustandekommen der Kette realisiert würden. Allerdings müssten die in diesem Modell fehlenden Anforderungen der Neutralität und des Interessenausgleichs kompensiert werden, beispielsweise indem der Wärmenetzbetrieb reguliert wird, sodass diese Monopolstellung nicht auf Kosten der angeschlossenen Abnehmer geht. Realistisch betrachtet, ist dieses Modell eher als Business-to-BusinessModell vorstellbar: Entweder es kommen in überschaubaren Zeiträumen interessante betriebswirtschaftliche Bedingungen zustande - oder eben nicht.

Schließlich ist auch möglich, dass gemeinwohlorientierte zivilgesellschaftliche Akteure, wie beispielsweise Lokale Agenda 21-Gruppen oder Bürgerstiftungen, eine RePro-Kette initiieren und vorantreiben. Das Neutralitätsgebot wäre erfüllt, die lokale Verankerung kann in den meisten Fällen unterstellt werden. Schwächen haben solche Initiativen häufig, zumindest zu Beginn, in der unternehmerischen Denke. Die gelegentliche Überbewertung von (partikularen, zum Beispiel ökologischen) Gemeinwohl- gegenüber Individualinteressen kann unter Umständen hinderlich sein. Dagegen ist Zähigkeit - und gelegentlich auch Charisma - meistens vorhanden.

\section{Transaktionskosten - ein lösbares Problem?}

RePro-Ketten sind eine regionalwirtschaftlich interessante Variante für den Aufbau dezentraler Versorgungsstrukturen. Dabei lohnt neben der Betrachtung der beteiligten Akteure auch die Analyse der mit dem wirtschaftlichen Austausch einhergehenden Kostengröße, den Transaktionskosten.

\section{Transaktionskosten als Hemmnis}

Transaktionskosten sind eine nur bedingt quantifizierbare Größe, die nichtsdestotrotz enorme Wichtigkeit für den Realisierungsprozess der RePro-Ketten hat. Grundsätzlich beschreiben sie eine Art „Reibungskosten“ des wirtschaftlichen Systems und entstehen beim Transfer eines Gutes oder einer Dienstleistung über eine technisch separierbare Schnittstelle (Williamson 1985). Entsprechend dieser Definition wird eine Reihe von Kostenarten unter dem Begriff der Transaktionskosten subsumiert: Anbahnungskosten, die die Kosten der Informationssuche und -beschaffung zu potenziellen Transaktionspartnern und deren Konditionen abbilden, Vereinbarungskosten, die die Kosten für Verhandlungen, Vertragsformulierung und Einigung beinhalten, Kontrollkosten zur Sicherstellung der Einhaltung von Vereinbarungen sowie etwaig anfallende Anpassungskosten im Falle veränderter Bedingungen und mögliche Beendigungskosten (Picot 1982).
Im Kontext der RePro-Ketten findet an verschiedenen Schnittstellen entlang des Wertschöpfungsprozesses eine Übertragung von Gütern oder Dienstleistungen statt, wie zum Beispiel zwischen Ressourcenlieferanten und -verwertern oder Energieproduzenten und -abnehmern. Folglich ist der Umgang mit den an diesen Reibungspunkten entstehenden Transaktionskosten entscheidend für die Realisierung einer RePro-Kette.

\section{Umgang mit Transaktionskosten}

Grundsätzlich verfolgt der Transaktionskostenansatz das Ziel, verschiedene Transaktionseigenschaften durch geeignete Koordinationsmechanismen zu reduzieren. So empfiehlt es sich zum Beispiel, bei geringer (Transaktions-) Spezifität je nach Ausprägung anderer Transaktionseigenschaften wie zum Beispiel der Transaktionshäufigkeit oder der Transaktionsunsicherheit eine Marktbeziehung oder marktnahe Mechanismen wie beispielsweise Verträge zu wählen. Bei hoher Spezifität sind hingegen stets unterschiedliche Formen hierarchischer Koordinationsmechanismen wie zum Beispiel Gemeinschaftsunternehmen oder sogar die vollständige Integration ratsam, um durch hierarchische Weisungsrechte mögliche Abweichungen von Vereinbarungen zu unterbinden und hierdurch Transaktionskosten zu reduzieren (Williamson 1991).

Das RePro-Management kann durch seine Arbeit einen Beitrag leisten, Transaktionskosten zu reduzieren und so den Realisierungsprozess von dezentralen Versorgungsstrukturen voranzutreiben: Durch seine lokalen Aktivitäten trägt es zunächst dazu bei, mögliche Keimzellen für RePro-Ketten zu identifizieren und zu aktivieren sowie darüber hinaus den Trägern dieser Keimzellen in der ersten Phase durch proaktive Informationsbereitstellung Potenziale und Verwertungsmöglichkeiten aufzuzeigen. Somit reduziert es die Informationskosten von Akteuren, die als Träger von Versorgungsstrukturen infrage kommen.

In einem weiteren Schritt kann das RePro-Management aufgrund seiner lokalen Kenntnisse Projektträgern Informationen zu und Kontakte zwischen möglichen RePro-Ketten-Beteiligten bereitstellen. Dies reduziert nicht nur die Informationskosten der Beteiligten, sondern senkt auch die Vertragskosten, indem das RePro-Management bei der Anbahnung von Wirtschaftsbeziehungen unterstützend eingreift oder sogar während der Vertragsverhandlungen als Moderator und neutraler Vermittler unterschiedliche Interessen zwischen den Parteien austariert.

Schließlich kann das RePro-Management auch dazu beitragen, Transaktionskosten, die nach Vertragsabschluss entstehen (wie beispielsweise Anpassungskosten), zu verringern: Indem die Öffentlichkeitsarbeit als zentrales Tätigkeitsfeld begriffen wird, verschiedene Interessengruppen (nach Rücksprache mit den Beteiligten) informiert und in den Entwicklungsprozess eingebunden werden und sich hierdurch mögliche Anpassungskosten reduzieren lassen.

Somit hat das RePro-Management mit seinen Aktivitäten einen signifikanten Einfluss auf die Höhe der Transaktionskosten und damit auf die Umsetzungschancen der RePro-Ketten. 


\section{Fazit}

Im zweiten Abschnitt wurden verschiedene Modelle eines RePro-Managements diskutiert. Unabhängig davon, wer den RePro-Manager gibt: Es muss Zeit und damit in den allermeisten Fällen auch Geld in Form von Personalmitteln investiert werden. Während im Fall des professionellen Dienstleisters und des Unternehmens (Modelle 2 und 3) im Laufe des Aufbauprozesses eine Kosten-Nutzen-Abschätzung und während des Betriebs der Kette eine Kosten-Nutzen-Rechnung gemacht werden kann, ist das in den Fällen eines kommunalen oder zivilgesellschaftlichen RePro-Managers nicht so einfach, da Kosten und Nutzen an verschiedenen Stellen anfallen und der Nutzen darüber hinaus nicht zwangsläufig in quantitativen Größen messbar ist.

Angesichts des (heute noch) aufwendigen RePro-Managements und der Transaktionskosten, die mit dem Aufbau der RePro-Ketten verbunden sind, muss man sich abschließend der Frage stellen: Lohnt sich das überhaupt?

Da die RePro-Ketten noch nicht in Betrieb sind, kann ihr Nutzen noch nicht erhoben werden. Auf die Frage, welche Vorteile RePro bietet, hat der Bürgermeister aber durchaus heute schon Antworten: „sichert Arbeitsplätze“, „junge Leute engagieren sich“, „regionale Wertschöpfung entsteht“, „es werden wieder Häuser gebaut“, „Versorgung wird langfristig gesichert“, „stabile Leistungen und Preise bei Wasser, Abwasser und Wärme“, „weniger Abhängigkeit von den Entscheidungen und Preisen großer Unternehmen, die nicht hier sitzen“, „und das alles umweltgerecht und nachhaltig“. Doch wie setzt man diesen - zum Teil prognostizierten - qualitativen Nutzen zu den quantitativen Kosten ins rechte Verhältnis? Diese Frage kann der RePro-Forschungsverbund nicht mehr beantworten. Doch um eine entsprechende Methode zur Entwicklung eines regionalen Wohlstandsradars für solche kleinen Public Private Partnerships, wie die RePro-Ketten sie darstellen, kümmert sich das Folgeprojekt. Bis dahin werden die RePro-Ketten in Uebigau-Wahrenbrück und Gräfenhainichen voraussichtlich in Betrieb sein und zusammen mit ähnlichen Public Private Partnerships empirische Daten für das regionale Wohlstandsradar liefern.

Auch das Zusammenspiel zwischen institutionellen Rahmenbedingungen und dem Handeln der Akteure sollte weiter im Fokus der Forschung bleiben: Dass soziale Verhaltensmuster wie Fairness oder Altruismus insbesondere im lokalen Betrachtungs- und Handlungsrahmen verstärkt Relevanz haben, ist in jüngeren Arbeiten aus dem Bereich der empirischen und experimentellen Wirtschaftsforschung belegt worden (Fehr/Schmidt 1999). Die Anwendung dieser Erkenntnisse und die daraus resultierende Aufgabenstellung zur Untersuchung der Wechselwirkungen von institutionellen Steuerungsmaßnahmen und dem Verhalten der beteiligten Akteure sind jedoch noch am Anfang. So können etablierte, zumeist monetäre Anreizmechanismen (zum Beispiel Kompensationszahlungen) möglicherweise dem Interesse von einigen Projektbe- teiligtem (insbesondere mit ideellem Motiv) zuwiderlaufen, da dies als eine Form der Bestechung wahrgenommen werden könnte. Das Resultat wäre in diesem Fall in letzter Konsequenz das Scheitern von Infrastrukturprojekten. Der RePro-Manager als moderierender Akteur zwischen den Interessengruppen leistet somit durch seine Arbeit einen Beitrag zur Informationsvermittlung und zum Interessenausgleich, ohne auf monetäre Anreize zurückzugreifen. Dies als alleinigen Schlüssel zur erfolgreichen Realisierung der Re-Produktionsketten sowie von dezentralen Versorgungsstrukturen im Allgemeinen zu benennen, wird der Komplexität des Realisierungsprozesses von Infrastrukturen jedoch nicht gerecht, denn unter bestimmten Bedingungen könnten die für das Szenario der ideellen Motive als ungeeignet eingeschätzten monetären Anreize der entscheidende Faktor für eine erfolgreiche Realisierung von Infrastrukturen sein, sodass die Frage nach Wechselwirkungen zwischen Steuerungsmechanismen und Akteursverhalten ebenfalls noch weiter untersucht werden muss, um den ReProManager effektiver und effizienter einzusetzen.

\section{Anmerkungen}

[1] Alle Informationen und Instrumente sind unter www.reproketten.de zugänglich.

\section{Literatur}

Biesecker, A./Hofmeister, S. (2006): Die Neuerfindung des Ökonomischen. Ein (re)produktionstheoretischer Beitrag zur Sozial-ökologischen Forschung. München, oekom.

Fehr, E./Schmidt, K. M. (1999): A Theory of Fairness, Competition, and Cooperation. In: The Quarterly Journal of Economics 114, 3/1999. S. 817868.

Picot, A. (1982): Transaktionskostenansatz in der Organisationstheorie: Stand der Diskussion und Aussagewert. In: Die Betriebswirtschaft 42, 1/1982. S. $267-284$.

Schön, S. (2010): 3.000 m Hindernislauf. Regionales Konstellationsmanagement zwischen theoretischen Erkenntnissen und praktischen Erfordernissen. In: Binas, E. (Hrsg.): Wozu Region? - Chancen und Probleme im Transformationsprozess sog. strukturschwacher Regionen. Frankfurt am Main, Peter Lang. S. 213-229.

Williamson, O. E. (1991): Comparative Economic Organization: The Analysis of Discrete Structural Alternatives. In: Administrative Science Quarterly 36, 2/1991. S. 269-296.

Williamson, O. E. (1985): The Economic Institutions of Capitalism. New York, Free Press.

\section{AUTOREN + KONTAKT}

Dr. Susanne Schön ist Mitbegründerin, Geschäftsführerin und wissenschaftliche Leiterin des inter 3 Instituts für Ressourcenmanagement.

inter $3 \mathrm{GmbH}$ - Institut für Ressourcenmanagement, Otto-Suhr-Allee 59, 10585 Berlin. Tel. +49 3034347452 ,

E-Mail: schoen@inter3.de, Internet: www.inter3.de

Dipl.-Kfm. Dipl.-Vw. Özgür Yildiz ist wissenschaftlicher Mitarbeiter am Fachgebiet für Umweltökonomie und Wirtschaftspolitik der TU Berlin.

Technische Universität Berlin, Straße des 17. Juni 135,
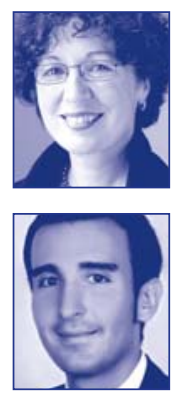
10623 Berlin. Tel. +4930 31425264, E-Mail: o.yildiz@tu-berlin.de 